CRYSTALLOGRAPHIC COMMUNICATIONS

ISSN 2056-9890

Received 28 April 2017

Accepted 23 May 2017

Edited by W. T. A. Harrison, University of Aberdeen, Scotland

Keywords: crystal structure; thiazolidine; thiophene; rhodanine; polythiophene.

CCDC reference: 1551679

Supporting information: this article has supporting information at journals.iucr.org/e

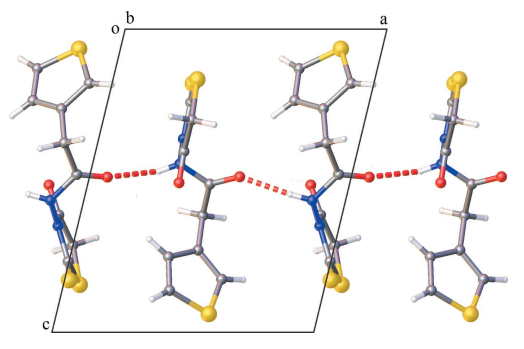

OPEN $\odot$ ACCESS

\section{Crystal structure of $\mathrm{N}$-(4-oxo-2-sulfanylidene-1,3- thiazolidin-3-yl)-2-(thiophen-3-yl)acetamide}

\author{
Trung Vu Quoc, ${ }^{\mathrm{a}}$ Linh Nguyen Ngoc, ${ }^{\mathrm{a}}$ Cong Nguyen Tien, ${ }^{\mathrm{b}}$ Chien Thang Pham ${ }^{\mathrm{c}}$ and \\ Luc Van Meervelt ${ }^{\mathrm{d}}$ *
}

\begin{abstract}
${ }^{\mathbf{a}}$ Faculty of Chemistry, Hanoi National University of Education, 136 Xuan Thuy, Cau Giay, Hanoi, Vietnam, ${ }^{\mathbf{b}}$ Faculty of Chemistry, Ho Chi Minh City University of Education, 280 An Duong Vuong Street, District No. 5, Ho Chi Minh City, Vietnam, 'Department of Chemistry, Hanoi University of Science, 19 Le Thanh Tong Street, Hoan Kiem District, Hanoi, Vietnam, and ${ }^{\mathbf{d}}$ Department of Chemistry, KU Leuven, Biomolecular Architecture, Celestijnenlaan 200F, Leuven (Heverlee), B-3001, Belgium. *Correspondence e-mail: luc.vanmeervelt@kuleuven.be
\end{abstract}

The title compound, $\mathrm{C}_{9} \mathrm{H}_{8} \mathrm{~N}_{2} \mathrm{O}_{2} \mathrm{~S}_{3}$, crystallizes with two molecules $(A$ and $B$ ) in the asymmetric unit. Both have similar conformations (overlay r.m.s. deviation = $0.209 \AA$ ) and are linked by an $\mathrm{N}-\mathrm{H}$. . O hydrogen bond. In both molecules, the thiophene rings show orientational disorder, with occupancy factors of 0.6727 (17) and 0.3273 (17) for molecule $A$, and 0.7916 (19) and 0.2084 (19) for molecule $B$. The five-membered rings make an angle of $79.7(2)^{\circ}$ in molecule $A$ and an angle of $66.8(2)^{\circ}$ in molecule $B$. In the crystal, chains of molecules running along the $a$-axis direction are linked by $\mathrm{N}-\mathrm{H} \cdots \mathrm{O}$ hydrogen bonds. The interaction of adjacent chains through $\mathrm{N}-\mathrm{H}$. . O hydrogen bonds leads to two types of ring structures containing four molecules and described by the graphset motifs $R_{4}^{4}(18)$ and $R_{4}^{2}(14)$.

\section{Chemical context}

Thiophene, $\mathrm{C}_{4} \mathrm{H}_{4} \mathrm{~S}$, belongs to a class of aromatic fivemembered heterocycles containing one $\mathrm{S}$ heteroatom. Thiophene and its derivatives occur in petroleum or coal (Orr \& White, 1990). Thiophene-based compounds have applications in modern drug design (Santagati et al., 1994), electronic and optoelectronic devices (Barbarella et al., 2005), and conductive and electroluminescent polymers (Friend et al., 1999). Also, several reviews of various aspects of thiophene coordination and reactivity in transition-metal complexes have been reported (Barbarella et al., 2005).<smiles>O=C(Cc1ccsc1)NN1C(=O)CSC1=S</smiles>

Derivatives of rhodanine (or 2-thioxo-1,3-thiazolidin-4one) have interesting pharmacological properties, such as the drug Epalrestat, which is an aldose reductase inhibitor used to treat diabetic neuropathy (Tomašić \& Mašič, 2012). Some other rhodanine derivatives were designed and synthesized for detecting tau pathology in the brains of patients with Alzheimer's disease (Ono et al., 2011).

As a continuation of our research (Nguyen et al., 2016; Vu et $a l ., 2016)$ on the chemical, physical and biological properties of new polythiophenes, a new thiophene monomer containing 
Table 1

Hydrogen-bond geometry $\left(\AA,^{\circ}\right)$.

$C g 1$ and $C g 2$ are the centroids of the $\mathrm{S} 1 A / \mathrm{C} 1 A-\mathrm{C} 4 A$ and $\mathrm{S} 11 A / \mathrm{C} 11 A-\mathrm{C} 14 A$ rings, respectively.

\begin{tabular}{lllll}
\hline$D-\mathrm{H} \cdots A$ & $D-\mathrm{H}$ & $\mathrm{H} \cdots A$ & $D \cdots A$ & $D-\mathrm{H} \cdots A$ \\
\hline $\mathrm{N} 1-\mathrm{H} 1 \cdots \mathrm{O} 11$ & $0.824(19)$ & $1.973(19)$ & $2.7923(16)$ & $173.1(18)$ \\
$\mathrm{N} 11-\mathrm{H} 11 \cdots \mathrm{O} 1^{\mathrm{i}}$ & $0.819(19)$ & $2.189(19)$ & $2.8436(16)$ & $137.1(16)$ \\
$\mathrm{N} 11-\mathrm{H} 11 \cdots \mathrm{O} 2^{\mathrm{ii}}$ & $0.819(19)$ & $2.519(18)$ & $3.0965(16)$ & $128.6(15)$ \\
$\mathrm{C} 5-\mathrm{H} 5 A \cdots \mathrm{O} 12^{\text {iii }}$ & 0.99 & 2.46 & $3.3901(19)$ & 156 \\
$\mathrm{C} 9-\mathrm{H} 9 A \cdots \mathrm{O} 2^{\text {iv }}$ & 0.99 & 2.53 & $3.2443(19)$ & 129 \\
$\mathrm{C} 9-\mathrm{H} 9 B \cdots \mathrm{S} 13^{\text {ii }}$ & 0.99 & 2.81 & $3.6570(17)$ & 144 \\
$\mathrm{C} 15-\mathrm{H} 15 A \cdots \mathrm{O} 2^{\text {ii }}$ & 0.99 & 2.37 & $3.2862(19)$ & 154 \\
$\mathrm{C} 9-\mathrm{H} 9 A \cdots C g 1^{\text {iv }}$ & 0.99 & 2.73 & $3.276(3)$ & 115 \\
$\mathrm{C} 19-\mathrm{H} 19 A \cdots C g 2^{\text {iii }}$ & 0.99 & 2.77 & $3.480(2)$ & 129 \\
\hline
\end{tabular}

Symmetry codes: (i) $x-1, y, z$; (ii) $-x+1,-y,-z+1$; (iii) $-x+1,-y+1,-z+1$; (iv) $-x+2,-y,-z+1$.

rhodanine has been prepared. In the presence of $\mathrm{FeCl}_{3}$, thiophene monomers can be polymerized by $\mathrm{C}-\mathrm{C}$ bond formation between the 2- and 5-positions of two subsequent thiophene monomers, resulting in an extended $\pi$-conjugated system. We present here the synthesis and crystal structure of $N$-(4-oxo-2-sulfanylidene-1,3-thiazolidin-3-yl)-2-(thiophen-3yl)acetamide, 3 .

\section{Structural commentary}

Crystals of the title compound belong to the triclinic space group $P \overline{1}$ with two independent molecules ( $A$ and $B$ ) per asymmetric unit (Fig. 1). In both molecules, the thiophene ring is disordered over two positions by a rotation of approximately $180^{\circ}$ around the $\mathrm{C} 5-\mathrm{C} 3$ or $\mathrm{C} 15-\mathrm{C} 13$ bond for molecules $A$ and $B$, respectively [occupancy factors $=0.6727$ (17) and 0.3273 (17) for molecule $A$, and $0.7916(19)$ and 0.2084 (19) for molecule $B$ ]. In the current discussion, only the major components will be considered. The 1,3-thiazolidine ring is almost planar (r.m.s. deviation $=0.020 \AA$ for ring S2/ N2/C7-C9 and $0.010 \AA$ for ring S12/N12/C17-C19) with the N3-substitiuents N1 [0.141 (1) ̊] and N11 [0.100 (1) A] situated in the same plane (deviations from plane given in parenthesis). Both thiophene rings are also planar as expected (r.m.s. deviation $=0.011 \AA$ for ring $\mathrm{S} 1 A / \mathrm{C} 1 A-\mathrm{C} 4 A$ and $0.002 \AA$ for ring $\mathrm{S} 11 A / \mathrm{C} 11 A-\mathrm{C} 14 A$ ), with the substituents $\mathrm{C} 5$

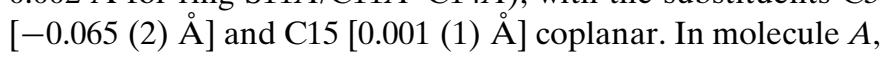
the heterocyclic rings make an angle of $79.7(2)^{\circ}$; in molecule $B$, this angle is $66.8(2)^{\circ}$. Also, the amide group and the 1,3 thiazolidine ring are oriented almost perpendicular to each other. In molecule $A$, the plane through the atoms of the amide group (N1/C6/O1) makes an angle of $76.32(8)^{\circ}$ with the best plane through the 1,3-thiazolidine ring; for molecule $B$, this angle is $83.88(6)^{\circ}$. Both molecules in the asymmetric unit are linked by an $\mathrm{N} 1-\mathrm{H} 1 \cdots \mathrm{O} 11$ hydrogen bond (Table 1 and Fig. 1).

\section{Supramolecular features}

The crystal packing is governed by hydrogen bonding. Chains of molecules are formed along the $a$-axis direction by alter-

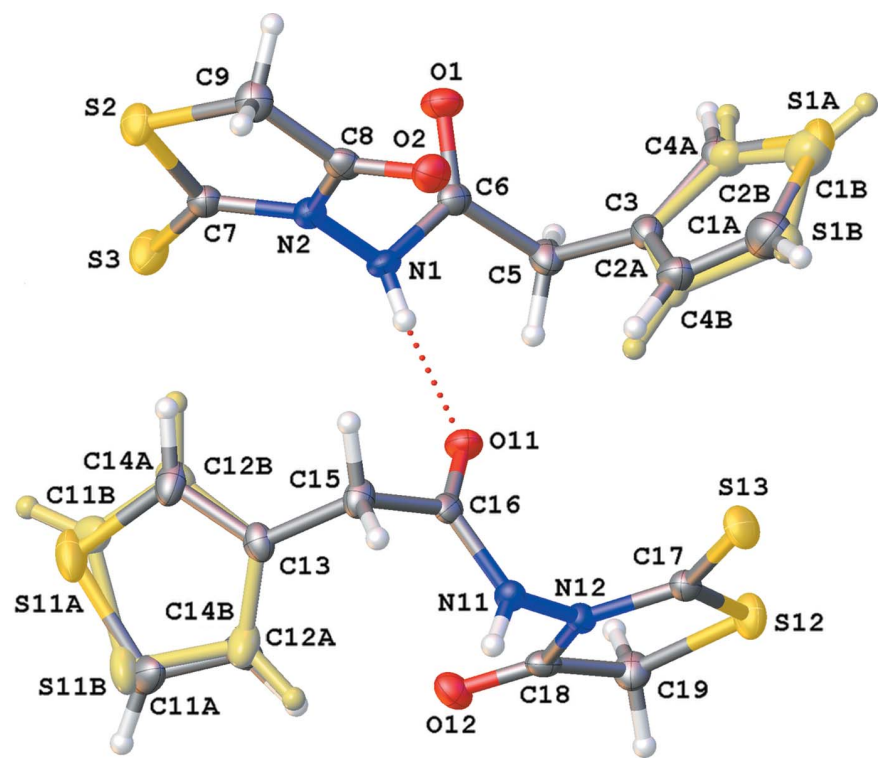

Figure 1

View of the asymmetric unit of the title compound, showing the atomlabelling scheme. Displacement ellipsoids are drawn at the $50 \%$ probability level. $\mathrm{H}$ atoms are shown as small circles of arbitrary radii. The minor component of the disordered thiophene rings is shown in pale yellow.

nating $\mathrm{N} 1-\mathrm{H} 1 \cdots \mathrm{O} 11$ and $\mathrm{N} 11-\mathrm{H} 11 \cdots \mathrm{O} 1$ hydrogen bonds (Table 1 and Fig. 2). The interaction of adjacent chains through $\mathrm{N} 11-\mathrm{H} 11 \cdots \mathrm{O} 2$ hydrogen bonds results in two different types of ring structures, each containing four molecules: (i) a ring structure of graph-set motif $R_{4}^{4}(18)$ showing also additional $\mathrm{C}-\mathrm{H} \cdots \mathrm{O}$ and $\mathrm{C}-\mathrm{H} \cdots \mathrm{S}$ interactions (Table 1 and Fig. 3), and (ii) a ring structure with graph-set motif $R_{4}^{2}(14)$ (Fig. 4). The packing shows a number of additional C$\mathrm{H} \cdots \mathrm{O}, \mathrm{C}-\mathrm{H} \cdots \mathrm{S}$ and weak $\mathrm{C}-\mathrm{H} \cdots \pi$ interactions (Table 1). The crystal packing contains no voids.

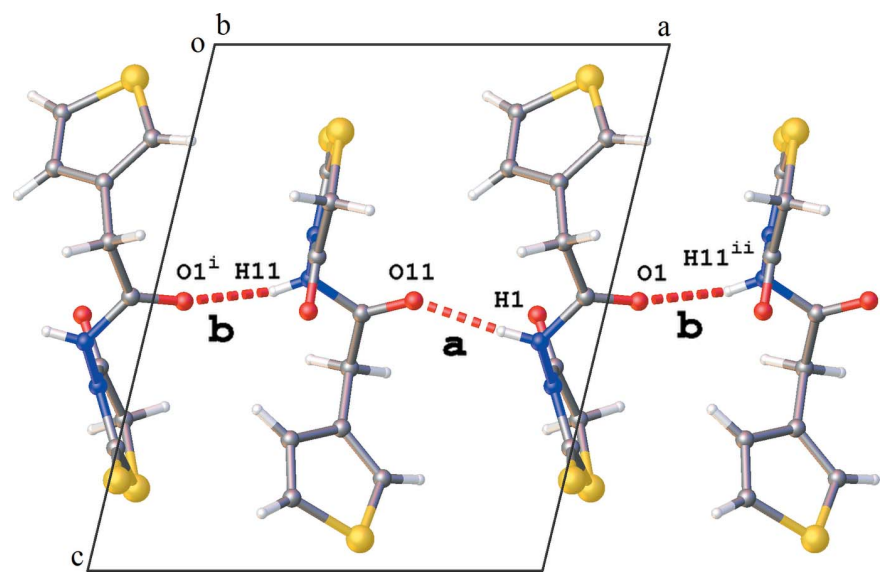

Figure 2

Part of the crystal packing of the title compound, showing a chain of molecules along the $a$ axis formed by $\mathrm{N}-\mathrm{H} \cdots \mathrm{O}$ hydrogen-bond interactions $\mathbf{a}$ and $\mathbf{b}$ [see Table 1; symmetry codes: (i) $x-1, y, z$; (ii) $x+1, y, z]$. 


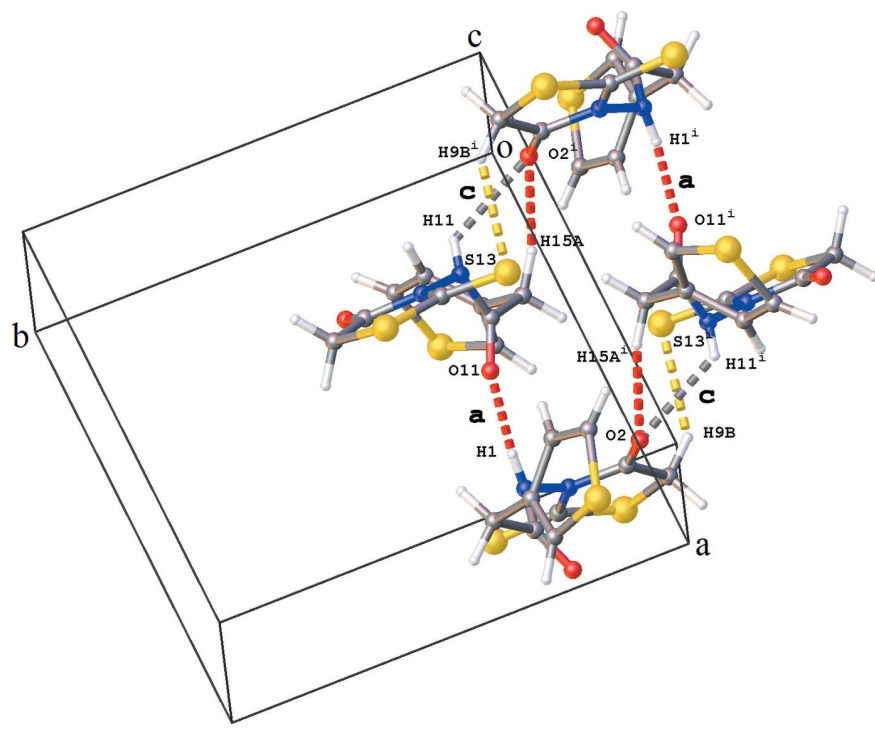

Figure 3

Ring of graph-set motif $R_{4}^{4}(18)$ formed by $\mathrm{N}-\mathrm{H} \cdots \mathrm{O}$ hydrogen-bond interactions a and $\mathbf{c}$ [see Table 1; symmetry code: (i) $-x+1,-y,-z+1$ ].

\section{Database survey}

A search of the Cambridge Structural Database (CSD, Version 5.38, last update February 2017; Groom et al., 2016) for structures containing an $N$-substituted 2-thioxo-1,3-thiazolidin-4-one ring gave 26 hits (169 hits when substituents at the 5-position are also allowed). In all cases, the 1,3-thiazolidine ring can be considered to be planar, as the largest deviation from the best plane through the ring atoms was only $0.070 \AA$ [for the complex bis(rhodanine)copper(I) iodide; refcode VICJUM; Moers et al., 1986]. The substituent at the $\mathrm{N} 3$ position is situated in the 1,3-thiazolidine plane, with a largest deviation of $0.174 \AA$ for the case with $-\mathrm{NH}_{2}$ as substituent (refcode EDEPUZ01; Jabeen et al., 2007).

Rotational disorder in $3-\mathrm{CH}_{2}$-thiophene fragments is frequently observed ( 25 structures of the 67 fragments present in the CSD).

\section{Synthesis and crystallization}

The reaction scheme to synthesize the title compound, $\mathbf{3}$, is given in Fig. 5.

\subsection{Synthesis of methyl 2-(thiophen-3-yl)acetate, 1}

Methyl thiophene-2-acetate, $1(5 \mathrm{mmol})$, was added to an excess of hydrazine hydrate $(40 \mathrm{mmol})$ in ethanol $(20 \mathrm{ml})$. The mixture was refluxed for $6 \mathrm{~h}$. The reaction mixture was

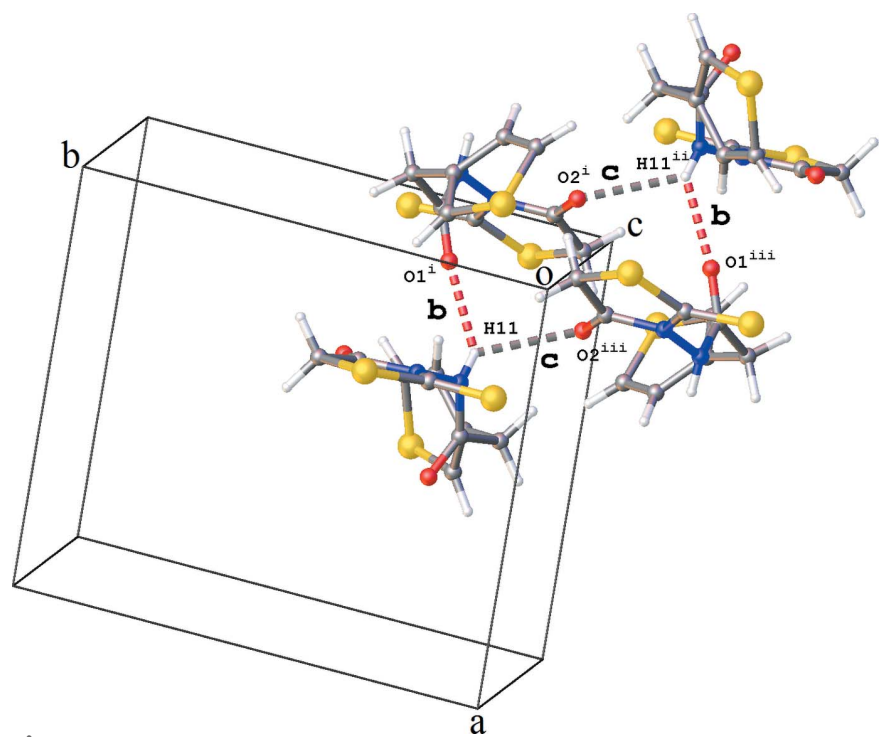

Figure 4

Ring of graph-set motif $R_{4}^{2}(14)$ formed by $\mathrm{N}-\mathrm{H} \cdots \mathrm{O}$ hydrogen-bond interactions $\mathbf{b}$ and $\mathbf{c}$ [see Table 1; symmetry codes: (i) $x-1, y, z$; (ii) $-x$, $-y,-z+1$; (iii) $-x+1,-y,-z+1]$.

allowed to cool. The resulting precipitate was filtered and recrystallized from ethanol solution to give $0.57 \mathrm{~g}$ (yield $74 \%$ ) of hydrazide 2 in the form of white crystals (m.p. $343 \mathrm{~K}$ ). IR (Nicolet Impact 410 FTIR, $\left.\mathrm{KBr}, \mathrm{cm}^{-1}\right)$ : 3323, $3068\left(v_{\mathrm{NH}}\right)$, 3068, $2957\left(v_{\mathrm{CH}}\right), 1641\left(v_{\mathrm{C}=\mathrm{O}}\right), 1526\left(v_{\mathrm{C}=\mathrm{C}}\right.$ thiophene $) .{ }^{1} \mathrm{H}$ NMR [Bruker XL-500, $500 \mathrm{MHz}, d_{6}$-DMSO, $\left.\delta(\mathrm{ppm}), J(\mathrm{~Hz})\right]$ : $7.22\left(d d, 1 \mathrm{H},{ }^{4} J=1.0,{ }^{5} J=2.0, \mathrm{H}^{2}\right), 7.01\left(d, 1 \mathrm{H},{ }^{5} J=5.0, \mathrm{H}^{4}\right)$, $7.43\left(d d, 1 \mathrm{H},{ }^{2} J=3.0,{ }^{4} J=4.5, \mathrm{H}^{5}\right), 3.32\left(s, 2 \mathrm{H}, \mathrm{H}^{6}\right), 9.14(s, 1 \mathrm{H}$, $\left.\mathrm{H}^{8}\right), 4.19\left(s, 2 \mathrm{H}, \mathrm{H}^{9}\right) .{ }^{13} \mathrm{C}$ NMR [Bruker XL-500, $125 \mathrm{MHz}, d_{6^{-}}$ DMSO, $\delta(\mathrm{ppm})$ ]: $122.06\left(\mathrm{C}^{2}\right), 135.95\left(\mathrm{C}^{3}\right), 128.62\left(\mathrm{C}^{4}\right), 125.59$ $\left(C^{5}\right), 35.10\left(C^{7}\right), 169.17\left(C^{8}\right)$. Calculation for $\mathrm{C}_{6} \mathrm{H}_{8} \mathrm{O}_{2} \mathrm{~N}_{2} \mathrm{~S}$ : $M=172 \mathrm{au}$.

\subsection{Synthesis of 2-(thiophen-3-yl)acetohydrazide, 2}

Methyl thiophene-2-acetate, $\mathbf{1}(5 \mathrm{mmol})$, was added to an excess of hydrazine hydrate $(40 \mathrm{mmol})$ in ethanol $(20 \mathrm{ml})$. The mixture was refluxed for $6 \mathrm{~h}$. The reaction mixture was allowed to cool. The resulting precipitate was filtered and recrystallized from ethanol solution to give $0.57 \mathrm{~g}$ (yield 74\%) of hydrazide 2 in the form of white crystals (m.p. $343 \mathrm{~K}$ ). IR (Nicolet Impact 410 FTIR, $\left.\mathrm{KBr}, \mathrm{cm}^{-1}\right)$ : 3323, $3068\left(v_{\mathrm{NH}}\right)$, 3068, $2957\left(v_{\mathrm{CH}}\right), 1641\left(v_{\mathrm{C}=\mathrm{O}}\right), 1526\left(v_{\mathrm{C}=\mathrm{C}}\right.$ thiophene $) .{ }^{1} \mathrm{H}$ NMR [Bruker XL-500, $500 \mathrm{MHz}, d_{6}$-DMSO, $\left.\delta(\mathrm{ppm}), J(\mathrm{~Hz})\right]$ : $7.22\left(d d, 1 \mathrm{H},{ }^{4} J=1.0,{ }^{5} J=2.0, \mathrm{H}^{2}\right), 7.01\left(d, 1 \mathrm{H},{ }^{5} J=5.0, \mathrm{H}^{4}\right)$, $7.43\left(d d, 1 \mathrm{H},{ }^{2} J=3.0,{ }^{4} J=4.5, \mathrm{H}^{5}\right), 3.32\left(s, 2 \mathrm{H}, \mathrm{H}^{6}\right), 9.14(s, 1 \mathrm{H}$,

Figure 5

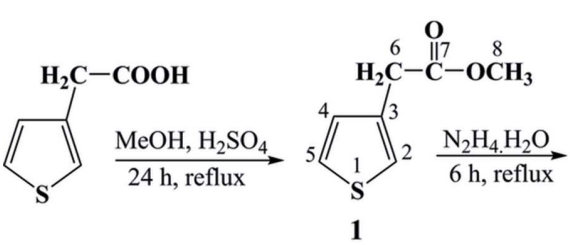

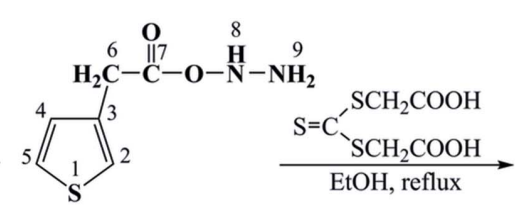

2

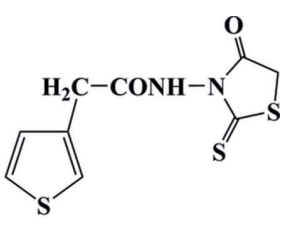

3

Reaction scheme for the title compound. 
$\left.\mathrm{H}^{8}\right), 4.19\left(s, 2 \mathrm{H}, \mathrm{H}^{9}\right) .{ }^{13} \mathrm{C}$ NMR [Bruker XL-500, $125 \mathrm{MHz}, d_{6-}$ DMSO, $\delta(\mathrm{ppm})$ ]: $122.06\left(\mathrm{C}^{2}\right), 135.95\left(\mathrm{C}^{3}\right), 128.62\left(\mathrm{C}^{4}\right), 125.59$ $\left(\mathrm{C}^{5}\right), 35.10\left(\mathrm{C}^{7}\right), 169.17\left(\mathrm{C}^{8}\right)$. Calculation for $\mathrm{C}_{6} \mathrm{H}_{8} \mathrm{O}_{2} \mathrm{~N}_{2} \mathrm{~S}: M=$ $172 \mathrm{au}$.

5.3. Synthesis of $N$-(4-oxo-2-sulfanylidene-1,3-thiazolidin-3yl)-2-(thiophen-3-yl)acetamide, 3

A mixture of hydrazide $2(10 \mathrm{mmol})$ and thiocarbonylbisthioglycolic acid $(10 \mathrm{mmol})$ in ethanol $(5 \mathrm{ml})$ was refluxed for $8 \mathrm{~h}$. After cooling, the resulting precipitate was filtered off, dried and recrystallized from ethanol solution to give $1.66 \mathrm{~g}$ (yield $61 \%$ ) of $\mathbf{3}$ as a pale-yellow crystals (m.p. $372 \mathrm{~K}$ ). IR (Nicolet Impact 410 FTIR, KBr, $\mathrm{cm}^{-1}$ ): 3442, 3292, 3226 $\left(v_{\mathrm{NH}}\right), 3148,2965,2921\left(v_{\mathrm{CH}}\right), 1727,1684\left(v_{\mathrm{C}=\mathrm{O}}\right), 1614,1532$ $\left(v_{\mathrm{C}}=\mathrm{C}\right.$ thiophene), 1244, $1177 \quad\left(v_{\mathrm{C}=\mathrm{S}}\right)$. Calculation for $\mathrm{C}_{9} \mathrm{H}_{8} \mathrm{O}_{2} \mathrm{~N}_{2} \mathrm{~S}_{3}: M=272$ au.

\section{Refinement}

Crystal data, data collection and structure refinement details are summarized in Table 2. Both thiophene rings are disordered over two positions by a rotation of approximately $180^{\circ}$ around the $\mathrm{C} 5-\mathrm{C} 3$ or $\mathrm{C} 15-\mathrm{C} 13$ bond for molecules $A$ and $B$, respectively. The final occupancy factors are 0.6727 (17) and 0.3273 (17) for molecule $A$, and 0.7916 (19) and 0.2084 (19) for molecule $B$. Bond lengths and angles in the disordered thiophene rings were restrained to target values derived from mean values observed in $3-\mathrm{CH}_{2}$-thiophene fragments in the CSD (Groom et al., 2016). The same anisotropic displacement parameters were used for equivalent atoms in the disordered thiophene rings (e.g. EADP $\mathrm{C} 1 A \mathrm{C} 1 B)$. The $\mathrm{H}$ atoms attached to atoms N1 and N11 were found in the difference density Fourier map and refined freely. The other $\mathrm{H}$ atoms were placed in idealized positions and refined in riding mode, with $U_{\text {iso }}(\mathrm{H})$ values assigned as $1.2 U_{\text {eq }}$ of the parent atoms, with $\mathrm{C}-\mathrm{H}$ distances of 0.95 (aromatic) and $0.99 \AA\left(\mathrm{CH}_{2}\right)$. In the final cycles of refinement, four outliers were omitted.

\section{Acknowledgements}

LVM thanks VLIR-UOS (project ZEIN2014Z182) for financial support.

\section{References}

Barbarella, G., Melucci, M. \& Sotgiu, G. (2005). Adv. Mater. 17, 15811593.

Bruker (2013). SAINT. Bruker AXS Inc., Madison, Wisconsin, USA. Bruker (2014). APEX2 and SADABS. Bruker AXS Inc., Madison, Wisconsin, USA.

Dolomanov, O. V., Bourhis, L. J., Gildea, R. J., Howard, J. A. K. \& Puschmann, H. (2009). J. Appl. Cryst. 42, 339-341.
Table 2

Experimental details.

\begin{tabular}{|c|c|}
\hline \multicolumn{2}{|l|}{ Crystal data } \\
\hline Chemical formula & $\mathrm{C}_{9} \mathrm{H}_{8} \mathrm{~N}_{2} \mathrm{O}_{2} \mathrm{~S}_{3}$ \\
\hline$M_{\mathrm{r}}$ & 272.35 \\
\hline Crystal system, space group & Triclinic, $P \overline{1}$ \\
\hline Temperature $(\mathrm{K})$ & 100 \\
\hline$a, b, c(\AA)$ & $9.6205(3), 10.8252(3), 11.5073(3)$ \\
\hline$\alpha, \beta, \gamma\left({ }^{\circ}\right)$ & $97.836(2), 102.720(2), 95.047(2)$ \\
\hline$V\left(\AA^{3}\right)$ & $1149.42(6)$ \\
\hline$Z$ & 4 \\
\hline Radiation type & Mo $K \alpha$ \\
\hline$\mu\left(\mathrm{mm}^{-1}\right)$ & 0.63 \\
\hline Crystal size $(\mathrm{mm})$ & $0.22 \times 0.07 \times 0.04$ \\
\hline \multicolumn{2}{|l|}{ Data collection } \\
\hline Diffractometer & Bruker APEXII CCD \\
\hline Absorption correction & $\begin{array}{l}\text { Multi-scan (SADABS; Bruker, } \\
\text { 2014) }\end{array}$ \\
\hline$T_{\min }, T_{\max }$ & $0.691,0.746$ \\
\hline $\begin{array}{l}\text { No. of measured, independent and } \\
\text { observed }[I>2 \sigma(I)] \text { reflections }\end{array}$ & $37647,6098,4985$ \\
\hline$R_{\text {int }}$ & 0.042 \\
\hline$(\sin \theta / \lambda)_{\max }\left(\AA^{-1}\right)$ & 0.682 \\
\hline \multicolumn{2}{|l|}{ Refinement } \\
\hline$R\left[F^{2}>2 \sigma\left(F^{2}\right)\right], w R\left(F^{2}\right), S$ & $0.032,0.077,1.03$ \\
\hline No. of reflections & 6098 \\
\hline No. of parameters & 323 \\
\hline No. of restraints & 40 \\
\hline $\mathrm{H}$-atom treatment & $\begin{array}{l}\mathrm{H} \text { atoms treated by a mixture of } \\
\text { independent and constrained } \\
\text { refinement }\end{array}$ \\
\hline$\Delta \rho_{\max }, \Delta \rho_{\min }\left(\mathrm{e} \AA^{-3}\right)$ & $0.38,-0.25$ \\
\hline
\end{tabular}

Computer programs: APEX2 (Bruker, 2014), SAINT (Bruker, 2013), SHELXS1997 (Sheldrick, 2008), SHELXL (Sheldrick, 2015) and OLEX2 (Dolomanov et al., 2009).

Friend, R. H., Gymer, R. W., Holmes, A. B., Burroughes, J. H., Marks, R. N., Taliani, C., Bradley, D. D. C., Dos Santos, D. A., Brédas, J. L., Lögdlund, M. \& Salaneck, W. R. (1999). Nature, 397, 121-128.

Groom, C. R., Bruno, I. J., Lightfoot, M. P. \& Ward, S. C. (2016). Acta Cryst. B72, 171-179.

Jabeen, S., Palmer, R. A., Potter, B. S., Helliwell, M., Dines, T. J. \& Chowdhry, B. Z. (2007). J. Chem. Crystallogr. 39, 151-156.

Moers, F. G., Smits, J. M. M. \& Beurskens, P. T. (1986). J. Crystallogr. Spectrosc. Res. 16, 101-106.

Nguyen, N. L., Tran, T. D., Nguyen, T. C., Duong, K. L., Pfleger, J. \& Vu, Q. T. (2016). Vietnam. J. Chem. 54, 259-263.

Ono, M., Hayashi, S., Matsumura, K., Kimura, H., Okamoto, Y., Ihara, M., Takahashi, R., Mori, H. \& Saji, H. (2011). ACS Chem. Neurosci. 2, 269-275.

Orr, W. L. \& White, C. M. (1990). Editors. Geochemistry of Sulfur in fossil fuels. Washington, DC: American Chemical Society.

Santagati, A., Modica, M., Santagati, M., Garuso, A. \& Cutuli, V. (1994). Pharmazie, 49, 64-65.

Sheldrick, G. M. (2008). Acta Cryst. A64, 112-122.

Sheldrick, G. M. (2015). Acta Cryst. C71, 3-8.

Tomašić, T. \& Mašič, L. P. (2012). Expert Opin. Drug Discov. 7, 549560.

Vu, Q. T., Nguyen, N. L., Duong, K. L. \& Pfleger, J. (2016). Vietnam. J. Chem. 54, 730-735. 


\section{supporting information}

Acta Cryst. (2017). E73, 901-904 [https://doi.org/10.1107/S2056989017007629]

Crystal structure of N-(4-oxo-2-sulfanylidene-1,3-thiazolidin-3-yl)-2-(thiophen-3-yl)acetamide

Trung Vu Quoc, Linh Nguyen Ngoc, Cong Nguyen Tien, Chien Thang Pham and Luc Van

Meervelt

Computing details

Data collection: APEX2 (Bruker, 2014); cell refinement: SAINT v8.34A (Bruker, 2013); data reduction: SAINT v8.34A (Bruker, 2013); program(s) used to solve structure: SHELXS1997 (Sheldrick, 2008); program(s) used to refine structure: SHELXL (Sheldrick, 2015); molecular graphics: Olex2 (Dolomanov et al., 2009); software used to prepare material for publication: Olex2 (Dolomanov et al., 2009).

N-(4-Oxo-2-sulfanylidene-1,3-thiazolidin-3-yl)-2-(thiophen-3-yl)acetamide

Crystal data

$\mathrm{C}_{9} \mathrm{H}_{8} \mathrm{~N}_{2} \mathrm{O}_{2} \mathrm{~S}_{3}$

$M_{r}=272.35$

Triclinic, $P \overline{1}$

$a=9.6205(3) \AA$

$b=10.8252(3) \AA$

$c=11.5073(3) \AA$

$\alpha=97.836(2)^{\circ}$

$\beta=102.720(2)^{\circ}$

$\gamma=95.047(2)^{\circ}$

$V=1149.42(6) \AA^{3}$

Data collection

Bruker APEX-II CCD diffractometer

$\varphi$ and $\omega$ scans

Absorption correction: multi-scan

(SADABS; Bruker, 2014)

$T_{\min }=0.691, T_{\max }=0.746$

37647 measured reflections

\section{Refinement}

Refinement on $F^{2}$

Least-squares matrix: full

$R\left[F^{2}>2 \sigma\left(F^{2}\right)\right]=0.032$

$w R\left(F^{2}\right)=0.077$

$S=1.03$

6098 reflections

323 parameters

40 restraints
$Z=4$

$F(000)=560$

$D_{\mathrm{x}}=1.574 \mathrm{Mg} \mathrm{m}^{-3}$

Mo $K \alpha$ radiation, $\lambda=0.71073 \AA$

Cell parameters from 9925 reflections

$\theta=3.1-30.6^{\circ}$

$\mu=0.63 \mathrm{~mm}^{-1}$

$T=100 \mathrm{~K}$

Block, colourless

$0.22 \times 0.07 \times 0.04 \mathrm{~mm}$

6098 independent reflections

4985 reflections with $I>2 \sigma(I)$

$R_{\text {int }}=0.042$

$\theta_{\text {max }}=29.0^{\circ}, \theta_{\min }=2.9^{\circ}$

$h=-13 \rightarrow 13$

$k=-14 \rightarrow 14$

$l=-15 \rightarrow 15$

Primary atom site location: structure-invariant direct methods

Hydrogen site location: mixed

$\mathrm{H}$ atoms treated by a mixture of independent and constrained refinement

$w=1 /\left[\sigma^{2}\left(F_{\mathrm{o}}^{2}\right)+(0.0367 P)^{2}+0.3868 P\right]$

where $P=\left(F_{\mathrm{o}}{ }^{2}+2 F_{\mathrm{c}}{ }^{2}\right) / 3$

$(\Delta / \sigma)_{\max }=0.001$ 
$\Delta \rho_{\max }=0.38$ e $\AA^{-3}$

$\Delta \rho_{\min }=-0.25$ e $\AA^{-3}$

Special details

Geometry. All esds (except the esd in the dihedral angle between two 1.s. planes) are estimated using the full covariance matrix. The cell esds are taken into account individually in the estimation of esds in distances, angles and torsion angles; correlations between esds in cell parameters are only used when they are defined by crystal symmetry. An approximate (isotropic) treatment of cell esds is used for estimating esds involving l.s. planes.

Fractional atomic coordinates and isotropic or equivalent isotropic displacement parameters $\left(\hat{A}^{2}\right)$

\begin{tabular}{|c|c|c|c|c|c|}
\hline & $x$ & $y$ & $z$ & $U_{\text {iso }} * / U_{\text {eq }}$ & Occ. $(<1)$ \\
\hline $\mathrm{C} 1 \mathrm{~A}$ & $0.7012(6)$ & $0.0875(6)$ & $0.1304(5)$ & $0.0435(14)$ & $0.6727(17)$ \\
\hline $\mathrm{H} 1 \mathrm{~A}$ & 0.627681 & 0.019090 & 0.098279 & $0.052^{*}$ & $0.6727(17)$ \\
\hline $\mathrm{C} 1 \mathrm{~B}$ & $0.8309(14)$ & $0.1396(12)$ & $0.0779(11)$ & $0.0435(14)$ & $0.3273(17)$ \\
\hline H1B & 0.865405 & 0.109102 & 0.009449 & $0.052^{*}$ & $0.3273(17)$ \\
\hline $\mathrm{C} 2 \mathrm{~A}$ & $0.7136(6)$ & $0.1688(5)$ & $0.2354(6)$ & $0.0292(11)$ & $0.6727(17)$ \\
\hline $\mathrm{H} 2 \mathrm{~A}$ & 0.648572 & 0.161127 & 0.286104 & $0.035^{*}$ & $0.6727(17)$ \\
\hline $\mathrm{C} 2 \mathrm{~B}$ & $0.8979(13)$ & $0.2418(14)$ & $0.1659(11)$ & $0.0292(11)$ & $0.3273(17)$ \\
\hline $\mathrm{H} 2 \mathrm{~B}$ & 0.982111 & 0.291811 & 0.161213 & $0.035^{*}$ & $0.3273(17)$ \\
\hline $\mathrm{C} 3$ & $0.83043(16)$ & $0.26481(14)$ & $0.26249(13)$ & $0.0229(3)$ & \\
\hline $\mathrm{C} 4 \mathrm{~A}$ & $0.9131(5)$ & $0.2528(5)$ & $0.1785(4)$ & $0.0196(7)$ & $0.6727(17)$ \\
\hline $\mathrm{H} 4 \mathrm{~A}$ & 0.998639 & 0.306140 & 0.183286 & $0.024 *$ & $0.6727(17)$ \\
\hline $\mathrm{C} 4 \mathrm{~B}$ & $0.7015(13)$ & $0.1835(11)$ & $0.2382(11)$ & $0.0196(7)$ & $0.3273(17)$ \\
\hline $\mathrm{H} 4 \mathrm{~B}$ & 0.634214 & 0.186979 & 0.287497 & $0.024 *$ & $0.3273(17)$ \\
\hline $\mathrm{C} 5$ & $0.87295(17)$ & $0.36183(14)$ & $0.37536(13)$ & $0.0244(3)$ & \\
\hline H5A & 0.786386 & 0.394860 & 0.394322 & $0.029 *$ & \\
\hline $\mathrm{H} 5 \mathrm{~B}$ & 0.937159 & 0.432803 & 0.362651 & $0.029^{*}$ & \\
\hline C6 & $0.94939(15)$ & $0.30218(12)$ & $0.47922(12)$ & $0.0195(3)$ & \\
\hline $\mathrm{C} 7$ & $0.99694(15)$ & $0.26354(14)$ & $0.76650(13)$ & $0.0219(3)$ & \\
\hline C8 & $0.91801(15)$ & $0.08293(13)$ & $0.61312(13)$ & $0.0211(3)$ & \\
\hline C9 & $0.99968(17)$ & $0.02374(14)$ & $0.71264(13)$ & $0.0255(3)$ & \\
\hline H9A & 1.082011 & -0.012463 & 0.688482 & $0.031^{*}$ & \\
\hline H9B & 0.936797 & -0.044373 & 0.731533 & $0.031^{*}$ & \\
\hline N1 & $0.87242(13)$ & $0.28734(11)$ & $0.56472(11)$ & $0.0205(2)$ & \\
\hline $\mathrm{H} 1$ & $0.784(2)$ & $0.2785(16)$ & $0.5452(15)$ & $0.023(4)^{*}$ & \\
\hline $\mathrm{N} 2$ & $0.92535(12)$ & $0.21256(11)$ & $0.64903(10)$ & $0.0200(2)$ & \\
\hline $\mathrm{O} 1$ & $1.06854(11)$ & $0.26997(10)$ & $0.48825(10)$ & $0.0263(2)$ & \\
\hline $\mathrm{O} 2$ & $0.85315(11)$ & $0.03015(10)$ & $0.51434(10)$ & $0.0263(2)$ & \\
\hline S1A & $0.84226(13)$ & $0.12988(10)$ & $0.06504(10)$ & $0.0260(2)$ & $0.6727(17)$ \\
\hline S1B & $0.6811(3)$ & $0.0788(3)$ & $0.1107(3)$ & $0.0260(2)$ & $0.3273(17)$ \\
\hline S2 & $1.06254(4)$ & $0.14561(4)$ & $0.84299(3)$ & $0.02640(9)$ & \\
\hline S3 & $1.01734(5)$ & $0.41087(4)$ & $0.82790(4)$ & $0.03324(10)$ & \\
\hline $\mathrm{C} 11 \mathrm{~A}$ & $0.4107(4)$ & $0.3665(5)$ & $0.8615(4)$ & $0.0348(9)$ & $0.7916(19)$ \\
\hline H11A & 0.353131 & 0.424722 & 0.890676 & $0.042 *$ & $0.7916(19)$ \\
\hline $\mathrm{C} 12 \mathrm{~A}$ & $0.3757(4)$ & $0.2893(6)$ & $0.7510(5)$ & $0.0260(8)$ & $0.7916(19)$ \\
\hline $\mathrm{H} 12 \mathrm{~A}$ & 0.287724 & 0.288656 & 0.693747 & $0.031^{*}$ & $0.7916(19)$ \\
\hline C11B & $0.6014(12)$ & $0.3318(14)$ & $0.9163(10)$ & $0.0348(9)$ & $0.2084(19)$ \\
\hline H11B & 0.675966 & 0.365869 & 0.985263 & $0.042 *$ & $0.2084(19)$ \\
\hline C12B & $0.6096(19)$ & $0.238(3)$ & $0.819(2)$ & $0.0260(8)$ & $0.2084(19)$ \\
\hline
\end{tabular}




$\begin{array}{llllll}\text { H12B } & 0.693246 & 0.198134 & 0.815566 & 0.031^{*} & 0.2084(19) \\ \text { C13 } & 0.48263(16) & 0.20962(13) & 0.72904(12) & 0.0209(3) & \\ \text { C14A } & 0.5992(5) & 0.2286(6) & 0.8247(5) & 0.0279(11) & 0.7916(19) \\ \text { H14A } & 0.680976 & 0.184997 & 0.826702 & 0.033^{*} & 0.7916(19) \\ \text { C14B } & 0.3780(19) & 0.274(3) & 0.7503(19) & 0.0279(11) & 0.2084(19) \\ \text { H14B } & 0.285834 & 0.266998 & 0.697131 & 0.033^{*} & 0.2084(19) \\ \text { C15 } & 0.46840(16) & 0.11790(13) & 0.61472(12) & 0.0216(3) & \\ \text { H15A } & 0.378555 & 0.059746 & 0.598778 & 0.026^{*} & \\ \text { H15B } & 0.550170 & 0.067657 & 0.623555 & 0.026^{*} & \\ \text { C16 } & 0.46640(14) & 0.19004(12) & 0.51108(12) & 0.0173(3) & \\ \text { C17 } & 0.31832(14) & 0.24127(13) & 0.24248(12) & 0.0192(3) & \\ \text { C18 } & 0.33768(14) & 0.41284(13) & 0.40333(13) & 0.0190(3) & \\ \text { C19 } & 0.33812(17) & 0.48314(13) & 0.29957(13) & 0.0247(3) & \\ \text { H19A } & 0.428495 & 0.540567 & 0.315257 & 0.030^{*} & \\ \text { H19B } & 0.256618 & 0.533625 & 0.288467 & 0.030^{*} & \\ \text { N11 } & 0.33329(13) & 0.20086(11) & 0.44504(10) & 0.0173(2) & \\ \text { H11 } & 0.261(2) & 0.1832(16) & 0.4689(15) & 0.025(4)^{*} & \\ \text { N12 } & 0.32383(12) & 0.28276(10) & 0.36245(10) & 0.0168(2) & \\ \text { O11 } & 0.57399(10) & 0.23773(10) & 0.48702(9) & 0.0240(2) & \\ \text { O12 } & 0.34760(11) & 0.45633(9) & 0.50671(9) & 0.0247(2) & \\ \text { S11A } & 0.58246(9) & 0.33791(8) & 0.93937(6) & 0.0371(2) & 0.7916(19) \\ \text { S11B } & 0.4286(6) & 0.3711(5) & 0.8833(4) & 0.0371(2) & 0.2084(19) \\ \text { S12 } & 0.32221(5) & 0.36801(4) & 0.16587(3) & 0.02777(9) & \\ \text { S13 } & 0.30889(4) & 0.09510(3) & 0.17993(3) & 0.02742(9) & \\ & & & & & \end{array}$

Atomic displacement parameters $\left(\AA^{2}\right)$

\begin{tabular}{lllllll}
\hline & $U^{11}$ & $U^{22}$ & $U^{33}$ & $U^{12}$ & $U^{13}$ & $U^{23}$ \\
\hline C1A & $0.037(2)$ & $0.057(3)$ & $0.038(3)$ & $0.0079(19)$ & $0.0078(18)$ & $0.012(2)$ \\
C1B & $0.037(2)$ & $0.057(3)$ & $0.038(3)$ & $0.0079(19)$ & $0.0078(18)$ & $0.012(2)$ \\
C2A & $0.0221(16)$ & $0.040(3)$ & $0.0266(15)$ & $0.0060(14)$ & $0.0066(12)$ & $0.0074(15)$ \\
C2B & $0.0221(16)$ & $0.040(3)$ & $0.0266(15)$ & $0.0060(14)$ & $0.0066(12)$ & $0.0074(15)$ \\
C3 & $0.0264(7)$ & $0.0246(7)$ & $0.0184(7)$ & $0.0087(6)$ & $0.0044(5)$ & $0.0041(5)$ \\
C4A & $0.0234(17)$ & $0.0195(14)$ & $0.0152(15)$ & $0.0055(11)$ & $0.0024(12)$ & $0.0019(10)$ \\
C4B & $0.0234(17)$ & $0.0195(14)$ & $0.0152(15)$ & $0.0055(11)$ & $0.0024(12)$ & $0.0019(10)$ \\
C5 & $0.0303(8)$ & $0.0230(7)$ & $0.0212(7)$ & $0.0101(6)$ & $0.0067(6)$ & $0.0028(5)$ \\
C6 & $0.0185(7)$ & $0.0173(6)$ & $0.0204(7)$ & $0.0017(5)$ & $0.0028(5)$ & $-0.0019(5)$ \\
C7 & $0.0181(7)$ & $0.0271(7)$ & $0.0210(7)$ & $0.0045(6)$ & $0.0049(5)$ & $0.0035(5)$ \\
C8 & $0.0162(6)$ & $0.0232(7)$ & $0.0246(7)$ & $0.0002(5)$ & $0.0080(5)$ & $0.0026(5)$ \\
C9 & $0.0300(8)$ & $0.0213(7)$ & $0.0251(7)$ & $0.0012(6)$ & $0.0061(6)$ & $0.0049(6)$ \\
N1 & $0.0147(6)$ & $0.0262(6)$ & $0.0206(6)$ & $0.0072(5)$ & $0.0024(5)$ & $0.0033(5)$ \\
N2 & $0.0177(6)$ & $0.0224(6)$ & $0.0195(6)$ & $0.0038(5)$ & $0.0034(4)$ & $0.0027(4)$ \\
O1 & $0.0170(5)$ & $0.0314(6)$ & $0.0313(6)$ & $0.0057(4)$ & $0.0068(4)$ & $0.0042(4)$ \\
O2 & $0.0220(5)$ & $0.0260(5)$ & $0.0268(5)$ & $-0.0008(4)$ & $0.0025(4)$ & $-0.0017(4)$ \\
S1A & $0.0297(4)$ & $0.0297(4)$ & $0.0182(3)$ & $0.0060(3)$ & $0.0044(2)$ & $0.0029(2)$ \\
S1B & $0.0297(4)$ & $0.0297(4)$ & $0.0182(3)$ & $0.0060(3)$ & $0.0044(2)$ & $0.0029(2)$ \\
S2 & $0.0324(2)$ & $0.02659(19)$ & $0.02008(18)$ & $0.00490(15)$ & $0.00387(14)$ & $0.00602(14)$ \\
S3 & $0.0405(2)$ & $0.02536(19)$ & $0.0278(2)$ & $0.01014(17)$ & $-0.00280(17)$ & $-0.00329(15)$
\end{tabular}




\begin{tabular}{lllllll} 
C11A & $0.0280(16)$ & $0.0542(18)$ & $0.029(2)$ & $0.0119(13)$ & $0.0103(14)$ & $0.0206(16)$ \\
C12A & $0.0257(11)$ & $0.029(2)$ & $0.0267(10)$ & $0.0055(10)$ & $0.0130(9)$ & $0.0047(11)$ \\
C11B & $0.0280(16)$ & $0.0542(18)$ & $0.029(2)$ & $0.0119(13)$ & $0.0103(14)$ & $0.0206(16)$ \\
C12B & $0.0257(11)$ & $0.029(2)$ & $0.0267(10)$ & $0.0055(10)$ & $0.0130(9)$ & $0.0047(11)$ \\
C13 & $0.0285(7)$ & $0.0181(6)$ & $0.0170(6)$ & $0.0015(5)$ & $0.0070(5)$ & $0.0039(5)$ \\
C14A & $0.0390(17)$ & $0.0221(15)$ & $0.0190(12)$ & $0.0073(14)$ & $-0.0020(12)$ & $0.0030(11)$ \\
C14B & $0.0390(17)$ & $0.0221(15)$ & $0.0190(12)$ & $0.0073(14)$ & $-0.0020(12)$ & $0.0030(11)$ \\
C15 & $0.0268(7)$ & $0.0170(6)$ & $0.0185(7)$ & $0.0021(5)$ & $0.0009(5)$ & $0.0018(5)$ \\
C16 & $0.0194(6)$ & $0.0150(6)$ & $0.0161(6)$ & $0.0041(5)$ & $0.0032(5)$ & $-0.0022(5)$ \\
C17 & $0.0172(6)$ & $0.0217(7)$ & $0.0184(6)$ & $0.0033(5)$ & $0.0042(5)$ & $0.0017(5)$ \\
C18 & $0.0144(6)$ & $0.0186(6)$ & $0.0237(7)$ & $0.0043(5)$ & $0.0048(5)$ & $0.0004(5)$ \\
C19 & $0.0307(8)$ & $0.0192(7)$ & $0.0242(7)$ & $0.0064(6)$ & $0.0048(6)$ & $0.0041(5)$ \\
N11 & $0.0161(6)$ & $0.0194(6)$ & $0.0174(5)$ & $0.0016(4)$ & $0.0053(4)$ & $0.0044(4)$ \\
N12 & $0.0178(5)$ & $0.0164(5)$ & $0.0162(5)$ & $0.0035(4)$ & $0.0042(4)$ & $0.0016(4)$ \\
O11 & $0.0159(5)$ & $0.0321(6)$ & $0.0250(5)$ & $0.0045(4)$ & $0.0059(4)$ & $0.0054(4)$ \\
O12 & $0.0264(5)$ & $0.0228(5)$ & $0.0248(5)$ & $0.0022(4)$ & $0.0098(4)$ & $-0.0029(4)$ \\
S11A & $0.0573(5)$ & $0.0334(3)$ & $0.0176(3)$ & $0.0122(3)$ & $0.0034(2)$ & $-0.0020(2)$ \\
S11B & $0.0573(5)$ & $0.0334(3)$ & $0.0176(3)$ & $0.0122(3)$ & $0.0034(2)$ & $-0.0020(2)$ \\
S12 & $0.0389(2)$ & $0.02616(19)$ & $0.01882(18)$ & $0.00436(16)$ & $0.00618(15)$ & $0.00631(14)$ \\
S13 & $0.0352(2)$ & $0.02192(18)$ & $0.02263(18)$ & $0.00242(15)$ & $0.00702(15)$ & $-0.00483(13)$ \\
& & & & & & \\
\hline
\end{tabular}

Geometric parameters $\left(\AA,{ }^{\circ}\right)$

\begin{tabular}{llll}
\hline $\mathrm{C} 1 \mathrm{~A}-\mathrm{H} 1 \mathrm{~A}$ & 0.9500 & $\mathrm{C} 5-\mathrm{C} 6$ & $1.514(2)$ \\
$\mathrm{C} 1 \mathrm{~B}-\mathrm{H} 1 \mathrm{~B}$ & 0.9500 & $\mathrm{C} 6-\mathrm{N} 1$ & $1.3730(18)$ \\
$\mathrm{C} 1 \mathrm{~A}-\mathrm{C} 2 \mathrm{~A}$ & $1.370(8)$ & $\mathrm{C} 6-\mathrm{O} 1$ & $1.2141(17)$ \\
$\mathrm{C} 2 \mathrm{~A}-\mathrm{H} 2 \mathrm{~A}$ & 0.9500 & $\mathrm{C} 7-\mathrm{N} 2$ & $1.3878(18)$ \\
$\mathrm{C} 1 \mathrm{~B}-\mathrm{C} 2 \mathrm{~B}$ & $1.395(13)$ & $\mathrm{C} 7-\mathrm{S} 2$ & $1.7339(15)$ \\
$\mathrm{C} 2 \mathrm{~B}-\mathrm{H} 2 \mathrm{~B}$ & 0.9500 & $\mathrm{C} 7-\mathrm{S} 3$ & $1.6303(15)$ \\
$\mathrm{C} 2 \mathrm{~B}-\mathrm{C} 3$ & $1.410(11)$ & $\mathrm{C} 8-\mathrm{C} 9$ & $1.494(2)$ \\
$\mathrm{C} 2 \mathrm{~A}-\mathrm{C} 3$ & $1.412(6)$ & $\mathrm{C} 8-\mathrm{N} 2$ & $1.3988(18)$ \\
$\mathrm{C} 4 \mathrm{~A}-\mathrm{H} 4 \mathrm{~A}$ & 0.9500 & $\mathrm{C} 8-\mathrm{O} 2$ & $1.2065(17)$ \\
$\mathrm{C} 4 \mathrm{~B}-\mathrm{H} 4 \mathrm{~B}$ & 0.9500 & $\mathrm{C} 9-\mathrm{H} 9 \mathrm{~A}$ & 0.9900 \\
$\mathrm{C} 4 \mathrm{~A}-\mathrm{S} 1 \mathrm{~A}$ & $1.707(4)$ & $\mathrm{C} 9-\mathrm{H} 9 \mathrm{~B}$ & 0.9900 \\
$\mathrm{C} 1 \mathrm{~A}-\mathrm{S} 1 \mathrm{~A}$ & $1.747(7)$ & $\mathrm{C} 9-\mathrm{S} 2$ & $1.8102(15)$ \\
$\mathrm{C} 4 \mathrm{~B}-\mathrm{S} 1 \mathrm{~B}$ & $1.690(11)$ & $\mathrm{N} 1-\mathrm{H} 1$ & $0.825(18)$ \\
$\mathrm{C} 1 \mathrm{~B}-\mathrm{S} 1 \mathrm{~B}$ & $1.672(11)$ & $\mathrm{N} 1-\mathrm{N} 2$ & $1.3874(16)$ \\
$\mathrm{C} 11 \mathrm{~A}-\mathrm{H} 11 \mathrm{~A}$ & 0.9500 & $\mathrm{C} 13-\mathrm{C} 14 \mathrm{~A}$ & $1.366(4)$ \\
$\mathrm{C} 11 \mathrm{~A}-\mathrm{C} 12 \mathrm{~A}$ & $1.378(6)$ & $\mathrm{C} 13-\mathrm{C} 14 \mathrm{~B}$ & $1.323(14)$ \\
$\mathrm{C} 12 \mathrm{~A}-\mathrm{H} 12 \mathrm{~A}$ & 0.9500 & $\mathrm{C} 13-\mathrm{C} 15$ & $1.5086(18)$ \\
$\mathrm{C} 11 \mathrm{~B}-\mathrm{H} 11 \mathrm{~B}$ & 0.9500 & $\mathrm{C} 15-\mathrm{H} 15 \mathrm{~A}$ & 0.9900 \\
$\mathrm{C} 11 \mathrm{~B}-\mathrm{C} 12 \mathrm{~B}$ & $1.430(16)$ & $\mathrm{C} 15-\mathrm{H} 15 \mathrm{~B}$ & 0.9900 \\
$\mathrm{C} 12 \mathrm{~B}-\mathrm{H} 12 \mathrm{~B}$ & 0.9500 & $\mathrm{C} 15-\mathrm{C} 16$ & $1.5096(19)$ \\
$\mathrm{C} 12 \mathrm{~A}-\mathrm{C} 13$ & $1.442(4)$ & $\mathrm{C} 16-\mathrm{N} 11$ & $1.3613(17)$ \\
$\mathrm{C} 12 \mathrm{~B}-\mathrm{C} 13$ & $1.395(15)$ & $\mathrm{C} 16-\mathrm{O} 11$ & $1.2190(17)$ \\
$\mathrm{C} 14 \mathrm{~A}-\mathrm{H} 14 \mathrm{~A}$ & 0.9500 & $\mathrm{C} 17-\mathrm{N} 12$ & $1.3802(17)$ \\
$\mathrm{C} 14 \mathrm{~B}-\mathrm{H} 14 \mathrm{~B}$ & 0.9500 & $\mathrm{C} 17-\mathrm{S} 12$ & $1.7310(14)$ \\
$\mathrm{C} 11 \mathrm{~A}-\mathrm{S} 11 \mathrm{~A}$ & $1.773(4)$ & $\mathrm{C} 17-\mathrm{S} 13$ & $1.6326(14)$ \\
& & &
\end{tabular}




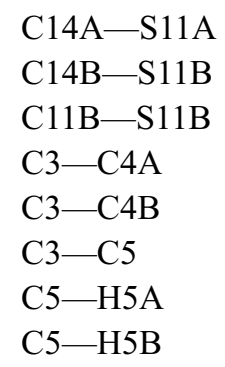

$1.694(4)$

$1.680(15)$

$1.726(12)$

$1.380(5)$

1.407 (12)

1.509 (2)

0.9900

0.9900

$92.8(2)$

125.5

125.5

125.0

125.0

122.6

122.7

114.8 (4)

$114.5(8)$

124.2

94.1 (5)

124.1

109.0 (4)

$109.9(7)$

93.1 (2)

126.1

126.1

122.6

$94.8(6)$

127.0

127.0

123.3

114.9 (3)

123.6

124.3

107.8 (3)

$106.0(8)$

$111.8(2)$

$109.4(6)$

$122.36(18)$

120.4 (4)

$125.5(2)$

130.2 (4)

109.8

109.8

$111.3(3)$

$114.2(8)$

$124.3(2)$

$122.8(6)$

$$
\begin{aligned}
& \mathrm{C} 18-\mathrm{C} 19 \\
& \mathrm{C} 18-\mathrm{N} 12 \\
& \mathrm{C} 18-\mathrm{O} 12 \\
& \mathrm{C} 19-\mathrm{H} 19 \mathrm{~A} \\
& \mathrm{C} 19-\mathrm{H} 19 \mathrm{~B} \\
& \mathrm{C} 19-\mathrm{S} 12 \\
& \mathrm{~N} 11-\mathrm{H} 11 \\
& \mathrm{~N} 11-\mathrm{N} 12
\end{aligned}
$$

$\mathrm{C} 8-\mathrm{C} 9-\mathrm{H} 9 \mathrm{~B}$

$\mathrm{C} 8-\mathrm{C} 9-\mathrm{S} 2$

$\mathrm{H} 9 \mathrm{~A}-\mathrm{C} 9-\mathrm{H} 9 \mathrm{~B}$

S2- C9-H9A

S2- C9-H9B

C6- $-\mathrm{N} 1-\mathrm{H} 1$

C6-N1-N2

$\mathrm{N} 2-\mathrm{N} 1-\mathrm{H} 1$

$\mathrm{C} 7-\mathrm{N} 2-\mathrm{C} 8$

$\mathrm{N} 1-\mathrm{N} 2-\mathrm{C} 7$

$\mathrm{N} 1-\mathrm{N} 2-\mathrm{C} 8$

C7-S2-C9

$\mathrm{C} 14 \mathrm{~B}-\mathrm{C} 13-\mathrm{C} 15$

C13-C15-H15A

$\mathrm{C} 3-\mathrm{C} 2 \mathrm{~A}-\mathrm{H} 2 \mathrm{~A}$

$\mathrm{C} 3-\mathrm{C} 2 \mathrm{~B}-\mathrm{H} 2 \mathrm{~B}$

$\mathrm{C} 3-\mathrm{C} 4 \mathrm{~A}-\mathrm{H} 4 \mathrm{~A}$

$\mathrm{C} 3-\mathrm{C} 4 \mathrm{~B}-\mathrm{H} 4 \mathrm{~B}$

C13-C15-H15B

$\mathrm{C} 3-\mathrm{C} 4 \mathrm{~A}-\mathrm{S} 1 \mathrm{~A}$

C3- $44 \mathrm{~B}-\mathrm{S} 1 \mathrm{~B}$

$\mathrm{C} 13-\mathrm{C} 15-\mathrm{C} 16$

H15A-C15-H15B

$\mathrm{C} 16-\mathrm{C} 15-\mathrm{H} 15 \mathrm{~A}$

C16-C15-H15B

N11-C16-C15

$\mathrm{O} 11-\mathrm{C} 16-\mathrm{C} 15$

$\mathrm{O} 11-\mathrm{C} 16-\mathrm{N} 11$

$\mathrm{N} 12-\mathrm{C} 17-\mathrm{S} 12$

$\mathrm{N} 12-\mathrm{C} 17-\mathrm{S} 13$

$\mathrm{S} 13-\mathrm{C} 17-\mathrm{S} 12$

N12-C18-C19

O12-C18-C19

O12-C18-N12

C18-C19-H19A

C18-C19-H19B

C18-C19-S12

H19A-C19-H19B

S12-C19-H19A
$1.501(2)$

$1.4073(17)$

$1.1982(16)$

0.9900

0.9900

$1.8121(15)$

0.819 (18)

1.3797 (16)

110.3

$107.29(10)$

108.5

110.3

110.3

$119.8(12)$

116.15 (11)

$112.4(12)$

$118.28(12)$

$121.89(12)$

$119.59(11)$

$93.85(7)$

$122.9(6)$

109.9

122.6

122.7

124.2

124.1

109.9

111.5 (2)

111.7 (6)

108.99 (11)

108.3

109.9

109.9

$115.08(12)$

123.93 (12)

120.98 (12)

109.87 (10)

125.87 (11)

$124.26(8)$

109.81 (11)

127.34 (13)

122.85 (13)

110.2

110.2

107.46 (10)

108.5

110.2 


\begin{tabular}{|c|c|c|c|}
\hline $\mathrm{C} 12 \mathrm{~A}-\mathrm{C} 13-\mathrm{C} 15$ & $124.4(2)$ & $\mathrm{S} 12-\mathrm{C} 19-\mathrm{H} 19 \mathrm{~B}$ & 110.2 \\
\hline $\mathrm{C} 3-\mathrm{C} 5-\mathrm{C} 6$ & $109.31(11)$ & $\mathrm{C} 16-\mathrm{N} 11-\mathrm{H} 11$ & $121.5(12)$ \\
\hline $\mathrm{H} 5 \mathrm{~A}-\mathrm{C} 5-\mathrm{H} 5 \mathrm{~B}$ & 108.3 & $\mathrm{C} 16-\mathrm{N} 11-\mathrm{N} 12$ & $117.50(11)$ \\
\hline $\mathrm{C} 6-\mathrm{C} 5-\mathrm{H} 5 \mathrm{~A}$ & 109.8 & N12-N11-H11 & $117.0(12)$ \\
\hline $\mathrm{C} 6-\mathrm{C} 5-\mathrm{H} 5 \mathrm{~B}$ & 109.8 & $\mathrm{C} 17-\mathrm{N} 12-\mathrm{C} 18$ & $118.85(11)$ \\
\hline $\mathrm{N} 1-\mathrm{C} 6-\mathrm{C} 5$ & $114.18(12)$ & $\mathrm{N} 11-\mathrm{N} 12-\mathrm{C} 17$ & $121.30(11)$ \\
\hline $\mathrm{O} 1-\mathrm{C} 6-\mathrm{C} 5$ & $123.98(13)$ & $\mathrm{N} 11-\mathrm{N} 12-\mathrm{C} 18$ & $119.39(11)$ \\
\hline $\mathrm{O} 1-\mathrm{C} 6-\mathrm{N} 1$ & $121.84(13)$ & $\mathrm{C} 17-\mathrm{S} 12-\mathrm{C} 19$ & $93.94(7)$ \\
\hline $\mathrm{N} 2-\mathrm{C} 7-\mathrm{S} 2$ & $109.78(10)$ & $\mathrm{C} 13-\mathrm{C} 12 \mathrm{~A}-\mathrm{H} 12 \mathrm{~A}$ & 122.6 \\
\hline $\mathrm{N} 2-\mathrm{C} 7-\mathrm{S} 3$ & $126.68(11)$ & $\mathrm{C} 13-\mathrm{C} 12 \mathrm{~B}-\mathrm{C} 11 \mathrm{~B}$ & $113.4(10)$ \\
\hline $\mathrm{S} 3-\mathrm{C} 7-\mathrm{S} 2$ & $123.53(9)$ & $\mathrm{C} 13-\mathrm{C} 12 \mathrm{~B}-\mathrm{H} 12 \mathrm{~B}$ & 123.3 \\
\hline $\mathrm{N} 2-\mathrm{C} 8-\mathrm{C} 9$ & $110.62(12)$ & $\mathrm{C} 13-\mathrm{C} 14 \mathrm{~A}-\mathrm{H} 14 \mathrm{~A}$ & 123.6 \\
\hline $\mathrm{O} 2-\mathrm{C} 8-\mathrm{C} 9$ & $126.72(14)$ & $\mathrm{C} 13-\mathrm{C} 14 \mathrm{~B}-\mathrm{H} 14 \mathrm{~B}$ & 124.3 \\
\hline $\mathrm{O} 2-\mathrm{C} 8-\mathrm{N} 2$ & $122.66(14)$ & $\mathrm{C} 13-\mathrm{C} 14 \mathrm{~A}-\mathrm{S} 11 \mathrm{~A}$ & $112.9(3)$ \\
\hline $\mathrm{C} 8-\mathrm{C} 9-\mathrm{H} 9 \mathrm{~A}$ & 110.3 & $\mathrm{C} 13-\mathrm{C} 14 \mathrm{~B}-\mathrm{S} 11 \mathrm{~B}$ & $111.5(9)$ \\
\hline $\mathrm{C} 2 \mathrm{~B}-\mathrm{C} 1 \mathrm{~B}-\mathrm{S} 1 \mathrm{~B}-\mathrm{C} 4 \mathrm{~B}$ & $0.0(13)$ & $\mathrm{N} 2-\mathrm{C} 8-\mathrm{C} 9-\mathrm{S} 2$ & $4.36(14)$ \\
\hline $\mathrm{C} 12 \mathrm{~A}-\mathrm{C} 11 \mathrm{~A}-\mathrm{S} 11 \mathrm{~A}-\mathrm{C} 14 \mathrm{~A}$ & $0.2(6)$ & $\mathrm{O} 1-\mathrm{C} 6-\mathrm{N} 1-\mathrm{N} 2$ & $-11.93(19)$ \\
\hline $\mathrm{C} 2 \mathrm{~A}-\mathrm{C} 1 \mathrm{~A}-\mathrm{S} 1 \mathrm{~A}-\mathrm{C} 4 \mathrm{~A}$ & $0.2(5)$ & $\mathrm{O} 2-\mathrm{C} 8-\mathrm{C} 9-\mathrm{S} 2$ & $-175.56(12)$ \\
\hline $\mathrm{C} 12 \mathrm{~B}-\mathrm{C} 11 \mathrm{~B}-\mathrm{S} 11 \mathrm{~B}-\mathrm{C} 14 \mathrm{~B}$ & $-2(2)$ & $\mathrm{O} 2-\mathrm{C} 8-\mathrm{N} 2-\mathrm{C} 7$ & $176.99(13)$ \\
\hline $\mathrm{S} 1 \mathrm{~B}-\mathrm{C} 1 \mathrm{~B}-\mathrm{C} 2 \mathrm{~B}-\mathrm{C} 3$ & $3.4(18)$ & $\mathrm{O} 2-\mathrm{C} 8-\mathrm{N} 2-\mathrm{N} 1$ & $-8.4(2)$ \\
\hline $\mathrm{S} 1 \mathrm{~A}-\mathrm{C} 1 \mathrm{~A}-\mathrm{C} 2 \mathrm{~A}-\mathrm{C} 3$ & $1.4(6)$ & $\mathrm{S} 2-\mathrm{C} 7-\mathrm{N} 2-\mathrm{C} 8$ & $-0.09(15)$ \\
\hline $\mathrm{S} 11 \mathrm{~B}-\mathrm{C} 11 \mathrm{~B}-\mathrm{C} 12 \mathrm{~B}-\mathrm{C} 13$ & $2(3)$ & $\mathrm{S} 2-\mathrm{C} 7-\mathrm{N} 2-\mathrm{N} 1$ & $-174.54(10)$ \\
\hline $\mathrm{S} 11 \mathrm{~A}-\mathrm{C} 11 \mathrm{~A}-\mathrm{C} 12 \mathrm{~A}-\mathrm{C} 13$ & $0.0(7)$ & $\mathrm{S} 3-\mathrm{C} 7-\mathrm{N} 2-\mathrm{C} 8$ & $-179.36(11)$ \\
\hline $\mathrm{C} 1 \mathrm{~B}-\mathrm{C} 2 \mathrm{~B}-\mathrm{C} 3-\mathrm{C} 4 \mathrm{~B}$ & $-5.7(17)$ & $\mathrm{S} 3-\mathrm{C} 7-\mathrm{N} 2-\mathrm{N} 1$ & $6.2(2)$ \\
\hline $\mathrm{C} 1 \mathrm{~A}-\mathrm{C} 2 \mathrm{~A}-\mathrm{C} 3-\mathrm{C} 4 \mathrm{~A}$ & $-2.9(7)$ & $\mathrm{S} 3-\mathrm{C} 7-\mathrm{S} 2-\mathrm{C} 9$ & $-178.31(10)$ \\
\hline $\mathrm{C} 1 \mathrm{~A}-\mathrm{C} 2 \mathrm{~A}-\mathrm{C} 3-\mathrm{C} 5$ & $-176.5(4)$ & $\mathrm{C} 12 \mathrm{~A}-\mathrm{C} 13-\mathrm{C} 15-\mathrm{C} 16$ & $65.3(4)$ \\
\hline $\mathrm{C} 1 \mathrm{~B}-\mathrm{C} 2 \mathrm{~B}-\mathrm{C} 3-\mathrm{C} 5$ & $177.4(9)$ & $\mathrm{C} 12 \mathrm{~B}-\mathrm{C} 13-\mathrm{C} 15-\mathrm{C} 16$ & $-106.2(18)$ \\
\hline $\mathrm{C} 2 \mathrm{~A}-\mathrm{C} 3-\mathrm{C} 5-\mathrm{C} 6$ & $76.8(3)$ & $\mathrm{C} 14 \mathrm{~A}-\mathrm{C} 13-\mathrm{C} 15-\mathrm{C} 16$ & $-114.1(4)$ \\
\hline $\mathrm{C} 4 \mathrm{~B}-\mathrm{C} 3-\mathrm{C} 5-\mathrm{C} 6$ & $84.8(6)$ & $\mathrm{C} 14 \mathrm{~B}-\mathrm{C} 13-\mathrm{C} 15-\mathrm{C} 16$ & 70.8 (19) \\
\hline $\mathrm{C} 4 \mathrm{~A}-\mathrm{C} 3-\mathrm{C} 5-\mathrm{C} 6$ & $-96.2(4)$ & $\mathrm{C} 13-\mathrm{C} 14 \mathrm{~B}-\mathrm{S} 11 \mathrm{~B}-\mathrm{C} 11 \mathrm{~B}$ & $2(2)$ \\
\hline $\mathrm{C} 11 \mathrm{~B}-\mathrm{C} 12 \mathrm{~B}-\mathrm{C} 13-\mathrm{C} 14 \mathrm{~B}$ & $0(3)$ & $\mathrm{C} 13-\mathrm{C} 14 \mathrm{~A}-\mathrm{S} 11 \mathrm{~A}-\mathrm{C} 11 \mathrm{~A}$ & $-0.4(5)$ \\
\hline $\mathrm{C} 11 \mathrm{~A}-\mathrm{C} 12 \mathrm{~A}-\mathrm{C} 13-\mathrm{C} 14 \mathrm{~A}$ & $-0.3(6)$ & $\mathrm{C} 13-\mathrm{C} 15-\mathrm{C} 16-\mathrm{N} 11$ & $-96.18(14)$ \\
\hline $\mathrm{C} 11 \mathrm{~A}-\mathrm{C} 12 \mathrm{~A}-\mathrm{C} 13-\mathrm{C} 15$ & $-179.8(4)$ & $\mathrm{C} 13-\mathrm{C} 15-\mathrm{C} 16-\mathrm{O} 11$ & $82.80(16)$ \\
\hline $\mathrm{C} 11 \mathrm{~B}-\mathrm{C} 12 \mathrm{~B}-\mathrm{C} 13-\mathrm{C} 15$ & $176.9(15)$ & $\mathrm{C} 15-\mathrm{C} 13-\mathrm{C} 14 \mathrm{~B}-\mathrm{S} 11 \mathrm{~B}$ & $-178.4(10)$ \\
\hline $\mathrm{C} 2 \mathrm{~A}-\mathrm{C} 3-\mathrm{C} 4 \mathrm{~A}-\mathrm{S} 1 \mathrm{~A}$ & $2.9(6)$ & $\mathrm{C} 15-\mathrm{C} 13-\mathrm{C} 14 \mathrm{~A}-\mathrm{S} 11 \mathrm{~A}$ & $179.9(2)$ \\
\hline $\mathrm{C} 2 \mathrm{~B}-\mathrm{C} 3-\mathrm{C} 4 \mathrm{~B}-\mathrm{S} 1 \mathrm{~B}$ & $5.5(12)$ & $\mathrm{C} 15-\mathrm{C} 16-\mathrm{N} 11-\mathrm{N} 12$ & $169.47(11)$ \\
\hline $\mathrm{C} 2 \mathrm{~B}-\mathrm{C} 3-\mathrm{C} 5-\mathrm{C} 6$ & $-98.6(10)$ & $\mathrm{C} 16-\mathrm{N} 11-\mathrm{N} 12-\mathrm{C} 17$ & $95.69(15)$ \\
\hline $\mathrm{C} 12 \mathrm{~A}-\mathrm{C} 13-\mathrm{C} 14 \mathrm{~A}-\mathrm{S} 11 \mathrm{~A}$ & $0.4(5)$ & $\mathrm{C} 16-\mathrm{N} 11-\mathrm{N} 12-\mathrm{C} 18$ & $-76.45(15)$ \\
\hline $\mathrm{C} 12 \mathrm{~B}-\mathrm{C} 13-\mathrm{C} 14 \mathrm{~B}-\mathrm{S} 11 \mathrm{~B}$ & $-1(3)$ & $\mathrm{C} 18-\mathrm{C} 19-\mathrm{S} 12-\mathrm{C} 17$ & $-0.76(11)$ \\
\hline $\mathrm{C} 3-\mathrm{C} 4 \mathrm{~A}-\mathrm{S} 1 \mathrm{~A}-\mathrm{C} 1 \mathrm{~A}$ & $-1.8(4)$ & $\mathrm{C} 19-\mathrm{C} 18-\mathrm{N} 12-\mathrm{C} 17$ & $2.19(17)$ \\
\hline $\mathrm{C} 3-\mathrm{C} 4 \mathrm{~B}-\mathrm{S} 1 \mathrm{~B}-\mathrm{C} 1 \mathrm{~B}$ & $-3.3(10)$ & $\mathrm{C} 19-\mathrm{C} 18-\mathrm{N} 12-\mathrm{N} 11$ & $174.53(11)$ \\
\hline $\mathrm{C} 3-\mathrm{C} 5-\mathrm{C} 6-\mathrm{N} 1$ & $-110.03(14)$ & $\mathrm{N} 12-\mathrm{C} 17-\mathrm{S} 12-\mathrm{C} 19$ & $1.91(11)$ \\
\hline $\mathrm{C} 3-\mathrm{C} 5-\mathrm{C} 6-\mathrm{O} 1$ & $70.25(18)$ & $\mathrm{N} 12-\mathrm{C} 18-\mathrm{C} 19-\mathrm{S} 12$ & $-0.57(14)$ \\
\hline $\mathrm{C} 5-\mathrm{C} 3-\mathrm{C} 4 \mathrm{~B}-\mathrm{S} 1 \mathrm{~B}$ & $-177.2(4)$ & $\mathrm{O} 11-\mathrm{C} 16-\mathrm{N} 11-\mathrm{N} 12$ & $-9.54(18)$ \\
\hline $\mathrm{C} 5-\mathrm{C} 3-\mathrm{C} 4 \mathrm{~A}-\mathrm{S} 1 \mathrm{~A}$ & $176.8(2)$ & $\mathrm{O} 12-\mathrm{C} 18-\mathrm{C} 19-\mathrm{S} 12$ & $179.28(12)$ \\
\hline $\mathrm{C} 5-\mathrm{C} 6-\mathrm{N} 1-\mathrm{N} 2$ & $168.34(12)$ & $\mathrm{O} 12-\mathrm{C} 18-\mathrm{N} 12-\mathrm{C} 17$ & $-177.67(13)$ \\
\hline $\mathrm{C} 6-\mathrm{N} 1-\mathrm{N} 2-\mathrm{C} 7$ & $104.36(15)$ & $\mathrm{O} 12-\mathrm{C} 18-\mathrm{N} 12-\mathrm{N} 11$ & $-5.33(19)$ \\
\hline
\end{tabular}




\begin{tabular}{llll}
$\mathrm{C} 6-\mathrm{N} 1-\mathrm{N} 2-\mathrm{C} 8$ & $-70.01(16)$ & $\mathrm{S} 12-\mathrm{C} 17-\mathrm{N} 12-\mathrm{C} 18$ & $-2.78(15)$ \\
$\mathrm{C} 8-\mathrm{C} 9-\mathrm{S} 2-\mathrm{C} 7$ & $-3.86(11)$ & $\mathrm{S} 12-\mathrm{C} 17-\mathrm{N} 12-\mathrm{N} 11$ & $-174.96(10)$ \\
$\mathrm{C} 9-\mathrm{C} 8-\mathrm{N} 2-\mathrm{C} 7$ & $-2.94(17)$ & $\mathrm{S} 13-\mathrm{C} 17-\mathrm{N} 12-\mathrm{C} 18$ & $177.43(10)$ \\
$\mathrm{C} 9-\mathrm{C} 8-\mathrm{N} 2-\mathrm{N} 1$ & $171.64(12)$ & $\mathrm{S} 13-\mathrm{C} 17-\mathrm{N} 12-\mathrm{N} 11$ & $5.25(19)$ \\
$\mathrm{N} 2-\mathrm{C} 7-\mathrm{S} 2-\mathrm{C} 9$ & $2.39(11)$ & $\mathrm{S} 13-\mathrm{C} 17-\mathrm{S} 12-\mathrm{C} 19$ & $-178.29(10)$ \\
\hline
\end{tabular}

Hydrogen-bond geometry $\left(A,{ }^{\circ}\right)$

$C g 1$ and $C g 2$ are the centroids of the $\mathrm{S} 1 \mathrm{~A} / \mathrm{C} 1 \mathrm{~A}-\mathrm{C} 4 \mathrm{~A}$ and $\mathrm{S} 11 \mathrm{~A} / \mathrm{C} 11 \mathrm{~A}-\mathrm{C} 14 \mathrm{~A}$ rings, respectively.

\begin{tabular}{lllll}
\hline$D-\mathrm{H} \cdots A$ & $D-\mathrm{H}$ & $\mathrm{H} \cdots A$ & $D \cdots A$ & $D-\mathrm{H} \cdots A$ \\
\hline $\mathrm{N} 1-\mathrm{H} 1 \cdots \mathrm{O} 11$ & $0.824(19)$ & $1.973(19)$ & $2.7923(16)$ & $173.1(18)$ \\
$\mathrm{N} 11-\mathrm{H} 11 \cdots \mathrm{O} 1^{\mathrm{i}}$ & $0.819(19)$ & $2.189(19)$ & $2.8436(16)$ & $137.1(16)$ \\
$\mathrm{N} 11-\mathrm{H} 11 \cdots \mathrm{O} 2^{\mathrm{ii}}$ & $0.819(19)$ & $2.519(18)$ & $3.0965(16)$ & $128.6(15)$ \\
$\mathrm{C} 5-\mathrm{H} 5 A \cdots \mathrm{O} 12^{\mathrm{iii}}$ & 0.99 & 2.46 & $3.3901(19)$ & 156 \\
$\mathrm{C} 9-\mathrm{H} 9 A \cdots \mathrm{O} 2^{\mathrm{iv}}$ & 0.99 & 2.53 & $3.2443(19)$ & 129 \\
$\mathrm{C} 9-\mathrm{H} 9 B \cdots \mathrm{S} 13^{\mathrm{ii}}$ & 0.99 & 2.81 & $3.6570(17)$ & 144 \\
$\mathrm{C} 15-\mathrm{H} 15 A \cdots \mathrm{O} 2^{\mathrm{ii}}$ & 0.99 & 2.37 & $3.2862(19)$ & 154 \\
$\mathrm{C} 9-\mathrm{H} 9 A \cdots C g 1^{\mathrm{iv}}$ & 0.99 & 2.73 & $3.276(3)$ & 115 \\
$\mathrm{C} 19-\mathrm{H} 19 A \cdots C g 2^{\mathrm{iii}}$ & 0.99 & 2.77 & $3.480(2)$ & 129
\end{tabular}

Symmetry codes: (i) $x-1, y, z$; (ii) $-x+1,-y,-z+1$; (iii) $-x+1,-y+1,-z+1$; (iv) $-x+2,-y,-z+1$. 